\title{
23. \\ Über eine Eigenschaft der Leitstrahlen der Kegelschnitte.
}

\author{
(Von Herrn Prof. J. Steiner zu Berlin.)
}

$E_{S \text { seien }} S, S_{1}$ die Scheitel der Haupt-Axe und $F^{\prime}, F_{1}$ die Brennpuncte eines Kegelschnitts; es seien ferner $\boldsymbol{P}, \boldsymbol{P}_{1}$ zwei solche Puncte in der Axe, welche zu den Scheiteln $\boldsymbol{S}, \boldsymbol{S}_{1}$ harmonisch sind, und zwar liege $\boldsymbol{S}$ zwischen $\boldsymbol{P}$ und $\boldsymbol{P}_{1}$; ferner liege $\boldsymbol{P}$ aufserhalb des Kegelschnitts, so dafs aus ihm zwei Tangenten $P T, P T_{1}$ an diesen gehen, deren Berührungssehne $\boldsymbol{T} \boldsymbol{T}_{1}$ die Axe im Puncte $\boldsymbol{P}_{1}$ trifft. Aus einem der Puncte $\boldsymbol{P}$ oder $\boldsymbol{P}_{1}$ ziehe man eine beliebige Secante $\boldsymbol{P A B}$ (oder $\boldsymbol{P}_{1} \boldsymbol{A B}$ ) durch den Kegelschnitt, und nach den Schnittpuncten $\boldsymbol{A}, \boldsymbol{B}$ ziehe man aus dem dem Scheitel $\boldsymbol{S}$ zunächst liegenden Brennuncte $\boldsymbol{F}$ die Leitstrahlen $\boldsymbol{F A}=\alpha, \boldsymbol{F B}=\boldsymbol{\beta}$, so wie endlich nach dem Berührungspuncte $\boldsymbol{T}$ den Leitstrahl $\boldsymbol{F} \boldsymbol{T}=\boldsymbol{\tau}$ : so giebt es jedesmal zwei bestimmte constante Gröfsen $r$ und $\boldsymbol{k}$ von der Beschaffenheit, dafs immer

$$
\text { 1. }(\alpha-r)(\beta-r)=(\tau-r)^{2}=k^{2},
$$

wie auch die Secante $\boldsymbol{A B}$ ihre Richtung und dadurch die Strahlen $\alpha$ und $\beta$ ihre Gröfse ändern mögen, und gleichviel ob die Secante durch $\boldsymbol{P}$ oder $\boldsymbol{P}_{1}$ gehen mag. Verãndert man aber die Lage der festen Pole $\boldsymbol{P}$ und $\boldsymbol{P}_{1}$, so ändern sich auch die Constanten $\boldsymbol{r}$ und $\boldsymbol{k}$.

Dem andern Brennpuncte $\boldsymbol{F}_{\mathfrak{l}}$ entspricht gleichzeitig die nämliche Constante $k$, dagegen eine andere Constante $r_{1}$, und wenn man die Leitstrahlen $\boldsymbol{F}_{1} \boldsymbol{A}=\alpha_{1}, \boldsymbol{F}_{1}^{\prime} \boldsymbol{B}=\beta_{1}, \boldsymbol{F}_{1} \boldsymbol{T}=\tau_{1}$ zieht, so hat man, wie für $\boldsymbol{F}$ :

$$
\left(\alpha_{1}-r_{1}\right)\left(\beta_{1}-r_{1}\right)=\left(\tau_{1}-r_{1}\right)^{2}=k^{2} \text {. }
$$

Ändern die conjugirten Pole $\boldsymbol{P}$ und $\boldsymbol{P}_{1}$ ihre Lage, so bleibt entweder die Summe oder der Unterschied der gleichzeitigen Gröfsen $\boldsymbol{r}$ und $\boldsymbol{r}_{\mathbf{1}}$ constant; nämlich diese Summe oder dieser Unterschied ist stets der Haupt-Axe 2 a des Kegelschnitts gleich, also

$$
\text { 2. } r_{1} \pm r=2 a \text {. }
$$


Bezeichnet man die Excentricilät des Kegelschnitts durch $c$, selzt die Tangente $\boldsymbol{P} \boldsymbol{T}=\boldsymbol{t}$ und den Winkel, welchen sie mit dem Leitstrahle $\boldsymbol{F T}=\boldsymbol{\tau}$ bildet, also den Winkel $\boldsymbol{P T F}=\varphi$, so hat man

$$
\begin{array}{ll}
\text { 3. } & k^{2}=(\boldsymbol{a}-\boldsymbol{r})^{2}-c^{2}, \\
\text { 4. } & k=\tau-r=\frac{1}{2} t \cdot \cos \varphi .
\end{array}
$$

Unter Umständen können von den Gröfsen $r, r_{1}, \alpha, \beta, \ldots$ einzelne ihr Vorzeichen ändern. Ich will dies, nebst einigen andern Besonderheiten, bei den verschiedenen Kegelschnitten etwas näher andeuten.

I. Bei der Ellipse kann die Gröfse $r$ positiv, negativ oder Null sein, je nachdem die Pole $\boldsymbol{P}$ und $\boldsymbol{P}_{1}$ liegen. Im letzten Fall, wo $\boldsymbol{r}=0$, wird die Gröfse $k$ der halben kleinen Axe $b$ der Ellipse gleich, so dafs für diesen Fall (1.)

$$
\text { 5. } \alpha \beta=\tau^{2}=b^{2} .
$$

Dies giebt den besonderen Satz:

„Beschreibt man mit der halben kleinen Axe b uin den Brennpunct $F$ der Ellipse einen Kreis, der die Ellipse allemal in swei reellen Puncten $T$ und $T_{1}$ scheidet, zieht die Sehne $T T_{1}$, die der Haupt-Axe in $P_{1}$ begegnet und legt in $T$ (oder $T_{1}$ ) an die Ellipse die Tangente TP, uelche die Haupt-Axe in $P$ Irifft, zieht ferner aus einem der Puncle $\boldsymbol{P}$ oder $\boldsymbol{P}_{1}$, gleichviel aus welchem, eine uillkürliche Secante $\boldsymbol{A B}$ durch die Ellipse und nach ihren Schnittpuncten $A$ und $B$ aus dem Brennpuncte $F$ die Strahlen $\alpha$ und $\beta$, so ist das Rechteck unter diesen Strahlen constant, und zwar gleich dem Quadrat über der halben kleinen Axe."

Rücken nun, von dem genannten Zustande ausgehend, die Pole $\boldsymbol{P}$ und $\boldsymbol{P}_{1}$ dem Scheitel $\boldsymbol{S}$ näher, so ist $\boldsymbol{r}$ positiv: entfernen sie sich dagegen von demselben, so wird $\boldsymbol{r}$ negativ und dann verwandeln sich die obigen Ausdrücke in folgende:

$$
\begin{array}{ll}
\text { 1. } & (\alpha+r)(\beta+r)=(\tau+r)^{2}=k^{2}, \\
\text { 2. } & r_{1}-r=2 a, \\
\text { 3. } & k^{2}=(a+r)^{2}-c^{2}, \\
\text { 4. } & k=\tau+r=\frac{1}{2} t \cdot \cos \varphi .
\end{array}
$$

Es sei $M$ der Mittelpunct der Ellipse und es werde $M P=p$ und $M P_{1}=p_{1}$ gesetzt, so finden ferner noch folgende Relationen statt: 
6. $\quad k=\frac{c}{2 a} \cdot \frac{p^{2}-a^{2}}{p}=\frac{c}{2 a} \cdot \frac{a^{2}-p_{1}^{2}}{p_{1}}=\frac{c}{2 a}\left(p-p_{1}\right)$,

7. $\quad r=a \mp \frac{c}{2 a} \cdot \frac{p^{2}+a^{2}}{p}=a \mp \frac{c}{2 a} \cdot \frac{p_{1}^{2}+a^{2}}{p_{1}}=a \mp \frac{c}{2 a}\left(p+p_{1}\right)$,

8. $p=\frac{a}{c}\left(k \pm \sqrt{ }\left(k^{2}+c^{2}\right)\right)=\frac{a}{c}\left[a-r \pm j\left((a-r)^{2}-c^{2}\right)\right]$,

wo die untern Zeichen in (7.) und (8.) den Werthen für $r_{1}$ und $p_{1}$ entsprechen.

II. Bei der Hyperbel sind die Gröfsen $r$ und $r_{1}$ immer positiv und für alle Lagen der Pole $\boldsymbol{P}$ und $\boldsymbol{P}_{1}$ ist immer

\section{2. $r_{1}-r=2 a$.}

In Rücksicht der Strahlen $\alpha, \beta$ kommt es dagegen darauf an, ob die Schnittpuncte $\boldsymbol{A}$ und $\boldsymbol{B}$ der Secante $\boldsymbol{A B}$ im nämlichen Zweige der Hyperbel liegen, oder nicht. Liegen sie im nämlichen $Z$ weige (der also $\boldsymbol{S}$ zum Scheitel hat und den Brennpunct $\boldsymbol{F}^{\prime}$ umschliefst), so hat man wie oben

$$
(\alpha-r)(\beta-r)=(\tau-r)^{2}=k^{2} \text { und }\left(\alpha_{1}-r_{1}\right)\left(\beta_{1}-r_{1}\right)=\left(\tau_{1}-r_{1}\right)^{2}=k^{2} \text {. }
$$

Dreht nun aber die Secante $\boldsymbol{A B}$ sich so um den festen Pol $\boldsymbol{P}$ oder $\boldsymbol{P}_{1}$, bis $\boldsymbol{B}$ sich ins Unendliche entfernt und von da in den andern $Z$ weig hinübergeht, so ändert der Strahl $\beta$ sein Zeichen, und damit wird nun gleichzeilig $\boldsymbol{r}>\boldsymbol{\alpha}$, während zuvor $\alpha>r$ war, so dafs alsdann die Formel in folgende übergeht: $(\boldsymbol{r}-\boldsymbol{\alpha})(\boldsymbol{r}+\boldsymbol{\beta})=(\tau-\boldsymbol{r})^{2}=\boldsymbol{k}^{2}$, und $\quad\left(\boldsymbol{r}_{1}-\boldsymbol{\alpha}_{1}\right)\left(\boldsymbol{r}_{1}+\boldsymbol{\beta}_{1}\right)=\left(\tau_{1}-\boldsymbol{r}_{1}\right)^{2}=\boldsymbol{k}^{2}$. Die Gröfsen $r$ und $r_{1}$ lassen sich hier auf eigenthümliche Art construiren. Aus einem der Pole $\boldsymbol{P}$ oder $\boldsymbol{P}_{1}$, etwa aus $\boldsymbol{P}$, ziehe man, einer Asymptote parallel, die Gerade $\boldsymbol{P R}$, welche die Hyperbel in $\boldsymbol{R}$ triff, und ziehe sodann die Leitstrahlen $\boldsymbol{F R}, \boldsymbol{F}_{1} \boldsymbol{R}$, 'so sind diese die verlangten Gröfsen $\boldsymbol{r}$ und $\boldsymbol{r}_{1}$.

III. Bei der Parabel sind die Pole $\boldsymbol{P}$ und $\boldsymbol{P}_{1}$ jedesmal gleich weit vom Scheitel $\boldsymbol{S}$ entfernt. Für alle Lagen dieser Pole bleibt die Gröfse $\boldsymbol{r}$ constant, und zwar ist sie stets der Entfernung des Brennpuncts vom Scheitel gleich, also ist $\boldsymbol{r}=\boldsymbol{F S}=\boldsymbol{e}$. Die Gröfse $\boldsymbol{k}$ ist jedesmal dem Abstande des Pols $\boldsymbol{P}$ oder $\boldsymbol{P}_{1}$ vom Scheitel $\boldsymbol{S}$ gleich, also $k=\boldsymbol{S P}=\boldsymbol{S P} \boldsymbol{P}_{1}=d$. Daher hat man:

$$
(\alpha-e)(\beta-e)=d^{2}
$$

d. h.: „Beschreibt man um den Brennpunct $F$ der Parabel mit dem Abstande e desselben voin Scheitel $\boldsymbol{S}$ einen Kreis, zieht sodann aus dem Brennpuncte nach irgend zwei Puncten $A$ und $B$ in der Parabel die Leitstrahlen $F A$ und $F B$, welche vom Kreise in $A_{1}$ und $B_{1}$ geschnitten werden, und zieht endlich die Sehne $A B$, welche die Parabel-Axe in 
340 25. Steiner, über cine Eigenschaft der Leitstrahlen der Kegelschnitte.

irgend einem Puncte $Q$ (d.i. $\boldsymbol{P}$ oder $\boldsymbol{P}_{1}$ ) trifft, so ist allemal das Rechteck unter denjenigen Abschnitten der Strahlen, welche zwischen dem Kreise und der Parabel liegen, gleich dem Quadrat über dem Abschnitle der Axe, welcher swischen ihrem Scheitel und dem Puncte $Q$ enthalten ist, also $\boldsymbol{A A}_{1} \cdot \mathrm{BB}_{1}=\boldsymbol{S Q ^ { 2 }}$."

Daraus schliefst man weiter den folgenden Satz:

„Schneiden eine beliebige Sehne $A B$ und die Tangenten in ihren Endpuncten $A$ und $B$ die Parabel-Axe beziehlich in $Q, A_{0}, B_{0}$, und trifft das aus dem gegenseitigen Schnittpuncle der Tangenten auf die Axe gefüllte Perpendikel dieselbe in $R$, so ist allemal

$$
\boldsymbol{S} \boldsymbol{A}_{0} \cdot S B_{0}=S Q^{2}=S R^{2} .
$$

Berlin, im April 1845. 